\title{
Mobile Location-based Awareness and Connectedness
}

\author{
Michael Voong \\ School of Computer Science \\ Birmingham, UK B15 2TT \\ +44 (0) 1214142790 \\ m.voong@cs.bham.ac.uk
}

\begin{abstract}
Mobile social awareness systems open up new social opportunities by enabling the automatic disclosure of awareness cues. We are trying to understand which cues work best to achieve feelings of connectedness between users and identify valid and comprehensive effect measures to evaluate such systems in HCI. We choose to study specifically two sensors location, via GPS and movement, via accelerometers.
\end{abstract}

The methodology of this research mixes in-lab testing with ethnographic studies using data to be collected using a selfreport, ecologically valid technique from psychology: ESM.

\section{Categories and Subject Descriptors}

H.1.2 [User/Machine Systems]: Human factors, H.5.m [Information Interfaces and Presentation (e.g., HCI)]: Miscellaneous

\section{General Terms}

Design, Human Factors

\section{Keywords}

Social awareness, location, deception, mobile, connectedness, design guidelines, experience sampling

\section{Introduction}

Awareness systems leverage sensor data by reprocessing, distributing, and representing them to others. Mobile awareness systems help create new opportunities for social interaction while on the move [9] by automatically broadcasting status information. Young people endure SMS even though it lacks expressiveness. However, it satisfies an important social and personal need to feel connected [11]. More contemporary systems broadcast location to aid social coordination. GPS sensing makes accurate disclosures convenient, but is attached to a whole host of privacy issues. Therefore, it is important to study how the data is stored and represented to users.

\section{Privacy of Location Disclosure}

Controlling location disclosure is becoming a very important issue as people become more self-conscious of how their location is perceived by systems and others.

When location used as a context-indicator to facilitate location-

(C) The Author 2008.

Published by the British Computer Society based search, there are little privacy issues as the data only flows between the service provider and the user. However, when locations are shared to others, the issue is more complex [1], raising issues of privacy, plausible deniability, uncertainty, self-perception and notions of place vs. activity [7, 8]. Indicating exact locations result in the surfacing of major privacy problems. Changing the representation to display a less granular location cue has been shown to reduce this problem, but may result in users feeling less in control [8].

The literature shows arguments that context-aware computing must leave interpretation to the user, as systems are unlikely to be able to infer human and social context in a reliable manner [2]. Schmandt and Marmasse discuss a system that encodes location data into something less directly interpretable [10], which is the visualization approach we are taking. Other research has shown that using mappings of location data - into a categorical representation of moving or not moving can often be enough, and can alleviate most privacy issues in a locationbased system aimed to communicate presence [3].

\section{Interim Work}

We have deployed a questionnaire $(\mathrm{N}=144)$ to users of Facebook, studying their location communications practices. This has given us insight into how technology should be appropriated to solve these problems. People take into account many factors when deciding what exactly to disclose, including current context, activity and nature of the request [4]. We found that deception commonly occurs, suggesting that location disclosure is more than just balancing privacy with utility - it includes a whole host of socio-technical problems where users are held accountable for their online representations. This is especially important to design for in automatic sensing settings as people will not always have a detailed awareness of what others can see.

The best way to visualize this may not be the immediately obvious, and widely used map-based representations, but a more abstract view that can potentially be simpler to read on a small screen and hide true locations when privacy is required. Our results showed higher levels of privacy concerns of mapbased visualizations of locations compared to relative visualizations. In line with this, one respondent to our survey said:

"A good idea would be a map that shows your friends location in relative geographical terms. If you enter the city centre the map would give a rough picture of their location. This could be say, friend A is situated in the Bullring or Friend B is at New Street station. A map giving the exact location of each friend is a little creepy in my opinion".

In order to gain further insight into the cognitive processes people go into when using location in traditional communication settings, a cultural probe was distributed on the Facebook platform, leveraging its mobile SMS capabilities. Users were asked to report on times when they use, or think of 
using current communications technology to contact others. They would note down details using SMS, or alternatively, using the website. This study has been important to verify whether the responses to the scenarios given in the questionnaire match with behavior in-situ. Outcomes include illustrations of cognitive drawings of the locations of others that favor relative location representations.

\section{RESEARCH QUESTIONS}

\section{RQ1: Visualization}

What is the favored visualization type for mobile social awareness systems?

Hypothesis: The favored visualization type will be of spatial nature, and involve visualization indicators of activity. Mapbased visualizations will not be favored due to representational complexity and conceptual difficulty, and the extra spatial detail is not required for effective communication of social context.

\section{RQ2: Effective Metrics}

Can we formulate an effective metrics and devise a relevant evaluation technique for connectedness and awareness?

In the literature we see a lack of comprehensive method for measuring connectedness. The research aims to generate valid measures from self-report questions and combine this with quantitative measures and other data to generate metrics.

Hypothesis: Self-report ESM questions that probe in-context the activities end mental states of participants, combined with usage activity logs are reliable indicators how successful mobile awareness systems are at raising group and individual awareness.

\section{RQ3: Group Awareness}

Can visualized location cues increase a sense of awareness and connectedness to groups and the individual?

Hypothesis: Knowing about the location of non-strangers increases feelings of connectedness and awareness of those people, and this can be appropriately measured and found to be statistically significant in longer trials.

\section{RQ4: Effect of Representation}

How does the representation of location and activity (accelerometer) affect the feelings of social presence and awareness/connectedness?

Hypothesis: Spatial, abstract, sub-symbolic representations create statistically similar measures of awareness and connectedness compared to absolute, map-based visualizations. More realistic motion visualizations will be a more powerful way of communicating bodily activity than simple ones.

\section{METHODOLOGY}

We are implementing an experimental platform on mobile phones and will first execute smaller lab-tests to ascertain preferred visualization approaches.

An experience sampling method-based design (ESM) will be applied for longer experiments, leveraging the mobile platform to gather quantitative and qualitative data on the effects of the system. An example qualitative question would be: "how confident are you that you know where [person] is right now?" or "how connected do you feel to your friends?", and quantitative: "how long has [name] spent in this location today?". Higher scores denote higher levels of connectedness. We can also evaluate preference of different visualizations by asking "How effective do you think the system is in representing the whereabouts of your contacts?".
A discussion of the validity of ESM can be found in the psychology literature [6] and its applications in evaluating ubiquitous computing in [5]: the "no-recall feature reduces the cognitive biases associated with other recall-based self-report techniques such as interviews, traditional surveys, and diaries".

The first three experiments will use ESM questions that work on finding the favored visualization method, accelerometer representation and privacy control method. The fourth experiment will focus on connectedness metrics for the chosen design in a longer-term, longitudinal ( 2 months) study, without intervention. It will be an ABA reversal design study with the application in use, with the intervention being the visualization. This design is used to isolate the cause-effect relationship of the system, whilst verifying how the system affects their perceptions of group connectedness over time.

\section{ACKNOWLEDGMENTS}

The author would like to thank his supervisor, Russell Beale.

\section{REFERENCES}

[1] Barkhuus, L. and Dey, A.K. 2003. Location-Based Services for Mobile Telephony: a Study of Users' Privacy Concerns. In Proceedings of Interact, 709-712.

[2] Bellotti, V. and Edwards, K. 2001. Intelligibility and accountability: Human Considerations in Context-aware Systems. Human-Computer Interaction, 16, 2-4, 193-212

[3] Bentley, F.R. and Metcalf, C.J. 2007. Sharing Motion Information with Close Family and Friends. In Proceedings of CHI '07 (New York, NY, USA) ACM Press, 1361-1370.

[4] Consolvo, S., Smith, I.E., Matthews, T., LaMarca, A., Tabert, J. and Powledge, P. 2005. Location Disclosure to Social Relations: Why, When, \& What People Want to Share. In Proceedings of CHI '05 (New York, NY, USA) ACM Press, 81-90.

[5] Consolvo, S. and Walker, M. 2003. Using the Experience Sampling Method to Evaluate Ubicomp Applications. IEEE Pervasive Computing, 2, 2, 24-31

[6] Csikszentmihalyi, M. and Larson, R. 1992. Validity and Reliability of the Experience Sampling Method. The Experience of Psychopathology: Investigating Mental Disorders in Their Natural settings, 43-57

[7] Girardin, F. 2007. Bridging the Social-technical Gap in Location-aware Computing. In Proceedings of CHI '07 (New York, NY, USA) ACM Press, 1653-1656.

[8] Iachello, G., Smith, I., Consolvo, S., Abowd, G.D., Hughes, J., Howard, J., Potter, F., Scott, J., Sohn, T., Hightower, J. and LaMarca, A. 2005. Control, Deception, and Communication: Evaluating the Deployment of a Location-enhanced Messaging service. In Proceedings of UbiComp 2005: Ubiquitous Computing, 213-231.

[9] Oulasvirta, A., Petit, R., Raento, M. and Tiitta, S. 2007. Interpreting and Acting on Mobile Awareness Cues. Human Computer Interaction, 22, 1 \& 2, 97-135

[10] Schmandt, C. and Marmasse, N. 2004. User-Centered Location Awareness. Computer, 37, 10, 110-111

[11] Vetere, F., Gibbs, M.R., Kjeldskov, J., Howard, S., Mueller, F.F., Pedell, S., Mecoles, K. and Bunyan, M. 2005. Mediating Intimacy: Designing Technologies to Support Strong-tie Relationships. In Proceedings of CHI '05 (New York, NY, USA) ACM Press, 471-480. 